\title{
$\beta$-catenin-mediated YAP signaling promotes human glioma growth
}

\author{
Yan Wang ${ }^{1,2+}$, Peng Pan ${ }^{3,4 \dagger}$, Zhaohao Wang ${ }^{3}$, Yu Zhang ${ }^{3}$, Peng Xie ${ }^{3}$, Decheng Geng ${ }^{3}$, Yang Jiang ${ }^{3}$, \\ Rutong $\mathrm{Yu}^{1,2,5}$ and Xiuping Zhou ${ }^{1,2,5^{*}}$
}

\begin{abstract}
Background: Hippo/YAP pathway is known to be important for development, growth and organogenesis, and dysregulation of this pathway leads to tumor progression.We and others find that YAP is up-regulated in human gliomas and associated with worse prognosis of patients. However, the role and mechanism of YAP in glioma progression is largely unknown.
\end{abstract}

Methods: The expression of YAP in glioma tissues was detected by quantitative polymerase chain reaction (qPCR) and immunoblotting. The effect of YAP on glioma progression was examined using cell growth assays and intracranial glioma model. The effect of YAP on $\beta$-catenin protein level, subcellular location and transcription activity was examined by immunoblotting, immunofluorescence and RT-PCR.

Results: Firstly, knockdown of YAP inhibited glioma cell proliferation in vitro and tumor growth in vivo. In addition, YAP modulated the protein level, subcellular location and transcription activity of $\beta$-catenin via regulating the activity of GSK3 $\beta$. Lastly, $\beta$-catenin partially mediated the effect of YAP on glioma cell proliferation.

Conclusion: Our findings identify that YAP promotes human glioma growth through enhancing Wnt/ $\beta$-catenin signaling. In addition, this study provides a new crosstalk mechanism between Hippo/YAP and Wnt/ $\beta$-catenin pathways, which suggests a new strategy for human glioma treatment.

Keywords: Glioma, Proliferation, YAP, $\beta$-catenin, GSK3 $\beta$, Nude mice

\section{Background}

Malignant glioma is the most common brain cancer. Affected patients are usually treated with a combined approach of surgery, chemotherapy and radiation therapy, but the median survival time is only $12-15$ months [1]. Therefore, understanding the molecular mechanism underlying pathogenesis of the disease is critical to identify specific molecular targets for glioma treatment.

Recent studies have identified the Hippo/YAP signaling as a key mechanism that controls organ size by imping on cell growth and proliferation [2, 3]. The MST1/2-WW45 complex phosphorylates and activates LATS1/2-MOB complex, which in turn phosphorylates oncogenic protein

\footnotetext{
* Correspondence: xpzhou@xzhmu.edu.cn

${ }^{\dagger}$ Equal contributors

${ }^{1}$ Insititute of Nervous System Diseases, Xuzhou Medical University, 84 West

Huai-hai Road, Xuzhou, Jiangsu 221002, People's Republic of China

${ }^{2}$ Brain Hospital, Affiliated Hospital of Xuzhou Medical University, Xuzhou,

Jiangsu, China

Full list of author information is available at the end of the article
}

YAP and TAZ. YAP and TAZ normally function in the nucleus as a co-activator for the TEAD/TEF family transcription factors to promote cell growth, proliferation, and survival. Phosphorylation of YAP and TAZ promotes their interaction with 14-3-3, leading to their cytoplasmic retention. Thus, the Hippo pathway activation inhibits transcriptional activity of YAP and TAZ [2, 3]. Accumulating evidence suggests that the Hippo/YAP pathway is dysregulated in many human cancers. Elevated YAP/TAZ expression or nuclear enrichment has been observed in many types of cancers, including liver, breast, lung, colon, ovary and others [4-6]. Orr et al. found that YAP1 is upregulated in the clinically aggressive glioblastoma subtypes (classical and mesenchymal) and is associated with the worst patient median survival [7]. Our pervious study also identified that YAP1/TAZ increased and meanwhile those of p-YAP1/p-TAZ and LATS1/2 decreased in gliomas. YAP1/TAZ-BIRC5 might be abnormally activated due to LATS1/2 down-regulation, which in turn promotes the 
occurrence and development of gliomas [8, 9]. Although YAP is up-regulated in gliomas and associated with worse prognosis of patients, the role and mechanism of YAP in gliomas is largely unknown.

The canonical Wnt pathway regulates many biological processes, including cell proliferation, cell fate decision, axis formation and organ development during embryonic development and tissue homeostasis [10, 11]. The key effector in this pathway is the transcriptional activator $\beta$-catenin. Without Wnt, cytoplasmic $\beta$-catenin is phosphorylated by GSK3 $\beta$ and then degraded. Upon Wnt stimulation, $\beta$-catenin is unphosphorylated, stabilized and then enters the nucleus to promote the transcription of downstream target genes, such as c-myc and cyclin D1. Dysregulation of Wnt/ $\beta$-catenin pathway has been implicated in a number of cancers [12]. Patients with high grade astrocytoma have a higher expression level of $\beta$-catenin and knockdown of $\beta$-catenin in human glioma cells inhibits cell proliferation and induces apoptosis [13-15].

Several studies have shown that the Hippo/YAP pathway genetically and functionally interacts with Wnt/ $\beta$-catenin signaling. For instance, Varelas et al. have shown that cytoplasmic TAZ binds to dishevelled and inhibits Wnt signaling [16]. Moreover, Heallen et al. reported that Hippo/YAP signaling inhibits $W n t / \beta$-catenin pathway in developing heart [17]. Later, Imajo et al. found that YAP and TAZ interact with $\beta$-catenin to block its nuclear localization, inhibiting Wnt/ $\beta$-catenin signaling [18]. However, Konsavage et al. reported that YAP is a direct $\mathrm{Wnt} / \beta$-catenin target gene and its expression is required for colorectal carcinoma cell growth [19]. In addition, Azzolin and his colleagues identified a mechanism that Wnt/ $/$-catenin signaling increases the level of YAP/TAZ [20]. Recently, Park et al. showed that the transcriptional regulators YAP/TAZ are found to be the key downstream effectors of alternative Wnt signaling, as well as negative regulation of canonical Wnt/ $\beta$-catenin signaling [21]. These reports suggest that the Hippo/YAP pathway and Wnt/ $\beta$-catenin signaling could regulate each other through multiple mechanisms, depending on biological contexts. Since dysregulation of Hippo/YAP and Wnt/ $\beta$-catenin pathways leads to tumor progression, elucidating the mutual regulatory mechanism of these two pathways might reveal potential targets for tumor therapeutic intervention.

Whether and how YAP regulates $\beta$-catenin to promote glioma cell proliferation remains poorly understood. In this study, we demonstrated that knockdown of YAP expression inhibited glioma cell proliferation in vitro and tumor growth in vivo. YAP modulated the protein level, subcellular location and transcription activity of $\beta$-catenin via regulating the activity of GSK3 $\beta$. At last, $\beta$-catenin mediated the effect of YAP on glioma cell proliferation. These results provide a novel mechanism that YAP promotes human glioma cell growth via $\beta$-catenin activation.

\section{Methods \\ Glioma and nontumor samples}

A total of 26 human glioma samples and 13 nontumor brain tissues (decompressive surgery) were obtained from Affiliated Hospital of Xuzhou Medical University. All of the glioma samples used in this study were astrocytomas, which were histologically diagnosed according to the World Health Organization grading system (12 of WHO grade II, 5 of WHO grade III, 3 of WHO grade IIIIV, 6 of WHO grade IV). All the glioma and nontumor brain tissues had been collected immediately after surgical resection and stored in $-80{ }^{\circ} \mathrm{C}$. Written informed consent was obtained from the patients and the study was approved by the Ethic Committee of the hospital.

\section{Cell culture}

The U87 and U251 glioma cells were purchased from Shanghai Cell Bank, Type Culture Collection Committee, Chinese Academy of Science. Cells were cultured in DMEM/F-12 (Gibco) media, supplemented with 10\% fetal bovine serum (FBS, BioInd, Israel).

\section{Antibodies and plasmids}

Antibodies against YAP1 were purchased from Abcam (Cambridge, MA). Antibodies specific for GSK3 $\beta$, pGSK3 $\beta$, $\beta$-catenin, Non-phospho active $\beta$-catenin (Ser33/ 37/Thr41), $\mathrm{p}-\beta$-catenin and GAPDH were obtained from Cell Signaling Technology (Beverly, MA). Antibody against Ki67 was purchased from Thermo. $\beta$-catenin ${ }^{C A}$ and $\beta$-catenin ${ }^{\mathrm{WT}}$ plasmids were kindly donated by Prof. Zhen-Ge Luo at the Institute of Neuroscience and Key Laboratory of Neurobiology, Chinese Academy of Sciences. YAP wild type and YAP S94A plasmids were kindly gifted by Prof. Bin Zhao at the Life Sciences Institute of Zhejiang University.

\section{Establishment of YAP down-regulation or over-expression glioma cells}

To establish YAP down-regulation glioma cells, short hairpin RNA (shRNA) targeting human YAP1 was inserted into lentiviral pLL3.7 backbone at HpaI and XhoI sites. For over-expression of YAP, the YAP2 cDNA was inserted into the pWPXLd backbone with GFP tag using PacI and MluI sites. The sequences (Sangon Biotech Shanghai) of shYAP were: F: $5^{\prime}$-TGCAGCAGAATATGATGAACTT CAAGAGAGTTCATCATATTCTGCTGCTTTTTTC-3'; R: 5' -TCGAGAAAAAAGCAGCAGAATATGATGAA CTCTCTTGAAGTTCATCATATT CTGCTGCA-3'. Primers sequences for YAP2 were: F: 5'-CCTTAAT TAAATGGATCCCGGGCAGCAG-3'; R: 5' -CGACGC GTCCCTATAACCATGTAAGAAAGC-3'. 


\section{RNA extraction, CDNA synthesis and quantitative PCR (qPCR)}

RNA was extracted from the human glioma cell lines U87 and U251 with YAP down-regulation or overexpression and the cDNA was synthesized using reverse transcription reagents (Roche, Basel, Switzerland) according to the manufacturer's protocol [8]. Quantitative PCR was performed on an ABI 7500 qPCR instrument (Applied Biosystems, Carlsbad, CA, USA) using SYBR Green. Primers are as follows: YAP-F, 5'-CACAGCTC AGCATCTTCGAC-3'; YAP-R, 5'-TATTCTGCTGCAC TGGTGGA-3'; $\beta$-catenin-F, 5'-CTTACACCCACCATC CCACT-3'; $\beta$-catenin-R, 5'-CCTCCACAAATTGCTGC TGT-3'; $\beta$-actin-F, 5'-CATGTACGTTGCTATCCAGG C-3'; $\beta$-actin-R, $5^{\prime}$-CTCCTTAATGTCACGCACGAT-3'.

\section{Cellular fractionation and Immunoblotting}

Cellular fractionation was conducted by using Membrane and Cytosol Protein Extraction Kit (Biovision), according to the instruction of manufacturer. Equal amount of protein lysates were subjected to $10 \%$ SDS-PAGE and then transferred to $0.45 \mu \mathrm{m}$ pore size PVDF membrane (Millipore). After blocking with 5\% non-fat milk, the membrane was probed with primary antibodies at $4{ }^{\circ} \mathrm{C}$ overnight and secondary antibodies at room temperature for $1 \mathrm{~h}$. Bound antibodies were detected by the Pierce ECL Plus Western Blotting Substrate (Thermo Fisher, Waltham, MA, USA) and exposed to X-ray films. Band densities were quantified by ImageJ Software (Wayne Rasband, National Institutes of Health, MD). The relative amount of proteins was determined by normalizing the densitometry value of interest to that of the loading control [22].

\section{EdU incorporation assay}

Cells were seeded into 96 -well plates at $7 \times 10^{3}$ cells per well. Twenty-four hours later, the cells were exposed to $50 \mu \mathrm{M}$ of 5-ethynyl-20-deoxyuridine (EdU; Ribobio, Guangzhou, China) for additional $2 \mathrm{~h}$ at $37{ }^{\circ} \mathrm{C}$. Then, the cells were fixed with $4 \%$ paraformaldehyde for $20 \mathrm{~min}$ and treated with $0.5 \%$ Triton-X-100 for another $20 \mathrm{~min}$ at room temperature. After being washed with PBS for five times, the cells were reacted with $100 \mu \mathrm{L}$ of $1 \times$ Apollo $^{\oplus}$ reaction cocktail for $30 \mathrm{~min}$. Thereafter, the DNA contents of cells were stained with $100 \mu \mathrm{L}$ of Hoechst 33,342 (5 $\mu \mathrm{g} / \mathrm{mL})$ for $20 \mathrm{~min}$ and visualized under a fluorescent microscope (IX71, Olympus, Tokyo, Japan). Data were obtained from three independent assays performed in triplicate [23].

\section{Cell counting Kit-8 assay}

Cell growth curves were obtained by detecting the cell viability with the Cell Counting Kit-8 (CCK-8, Dojindo, Japan) assay every $24 \mathrm{~h}$ according to the manufacturer's instruction.

\section{Colony formation assay}

Three days after lentivirus infection, cells were seeded into 6-well plate (300 cells per well). The medium was changed at three days intervals. After 14 days of culture at $37{ }^{\circ} \mathrm{C}$, the colonies were washed with PBS and fixed with $4 \%$ paraformaldehyde for $30 \mathrm{~min}$ at room temperature. The colonies were then stained with $0.05 \%$ crystal violet for $10 \mathrm{~min}$, washed with water and air-dried. The total number of colonies with more than 50 cells was counted.

\section{Intracranial model of glioma in nude mice}

Intracranial model of glioma in nude mice was performed according to our previous study [22]. All the in vivo experiments were carried out with ethical committee approval and met the standards required by the guidelines of Xuzhou Medical University. Female athymic nude mice aged 4-6 weeks and weighed $20 \mathrm{~g}$ were obtained from Experimental Animal Center of Xuzhou Medical University. Mice were randomly divided into two groups ( $n=6$ per group) and were anesthetized by intraperitoneal injection of $3 \%$ chloral hydrate $(0.01 \mathrm{~mL} /$ body weight $(\mathrm{g}))$. According to the coordinates, a small burr hole, $2 \mathrm{~mm}$ diameter, was drilled with $1.4 \mathrm{~mm}$ away from the midline at the right side of the cranium. The head of syringe was held in a horizontal position and shYAP or scramble U87 cells $\left(1 \times 10^{6}\right)$ were injected into right striatum at a depth of $2.6 \mathrm{~mm}$ using a small animal stereotactic apparatus. The cells were injected slowly within $10 \mathrm{~min}$ at a rate of $0.5 \mu \mathrm{L} / \mathrm{s}$ and the syringe stayed for $10 \mathrm{~min}$ before withdrawing. The burr hole was sealed with bone wax, strict aseptic conditions were applied in the whole experiments.

To obtain the survival curve, the mice were sacrificed when they appeared hemiplegia, listlessness, cachexia and other neurological symptoms or without neurological symptoms at 50 days after transplantation. The cryosections of brain were subjected to H.E. staining and the tumor volume was calculated according to the formula $V=1 / 2 a b^{2}$ with ' $a$ ' representing the longest diameter and 'b' representing the shortest diameter.

\section{Immunofluorescence}

Immunofluorescence was performed according to our previous study [22]. The brain sections containing the tumor or cells were incubated with $0.3 \%$ triton X-100 followed by $10 \%$ goat serum and then were exposed to a primary antibody (anti- $\beta$-catenin, anti-active- $\beta$-catenin and anti-Ki67) at $4{ }^{\circ} \mathrm{C}$ overnight. To visualize the positive cells, the sections or cells were incubated with Alexa fluor conjugated second antibody. The DAPI was used to stain cell nuclear. The sections were visualized under a fluorescent microscope (IX71 Olympus, Tokyo, Japan). 


\section{Statistical analysis}

Data were analyzed by using Student's $t$ test or ANOVA for multiple comparisons. In all analysis, quantitative data were obtained from at least three independent experiments and expressed as mean \pm SEM. $P$ values less than 0.05 were considered statistically significant $\left({ }^{*} P<0.05,{ }^{* * *} P<0.01,{ }^{* * * *} P<0.001\right)$.

\section{Results}

YAP is highly expressed in gliomas and knockdown of YAP inhibits glioma cell proliferation in vitro

In order to study the effect of YAP on glioma progression, we firstly examined the mRNA and protein level of YAP in human glioma tissues by real-time PCR and immunoblotting respectively. As shown in Fig. 1a-c, the mRNA and protein levels of YAP in human glioma tissues were significantly higher than that in nontumor tissues, in line with our previous report [8].
Therefore, we down-regulated YAP with shRNA lentivirus to examine the effect of YAP on glioma cell proliferation. As shown in supporting Fig. 1a and b (Additional file 1: Figure S1), the percentage of GFP positive cells (YAP knocking-down cells) was more than $95 \%$ and the downregulation efficiency was about $80 \%$ in both U251 and U87 cells. Examined by EdU incoporation assay and CCK-8 assay, depletion of YAP significantly inhibited the proliferation of U251 and U87 cells (Fig. 1d-g). Similarly, the number of the colonies formed from YAP down-regulation cells significantly decreased (Fig. 1h, i). The above results indicate that YAP down-regulation inhibited glioma cell proliferation in vitro.

\section{Down-regulation of YAP inhibits intracranial glioma growth in vivo}

To study the effect of YAP on intracranial glioma growth in vivo, YAP down-regulation U87 cells were

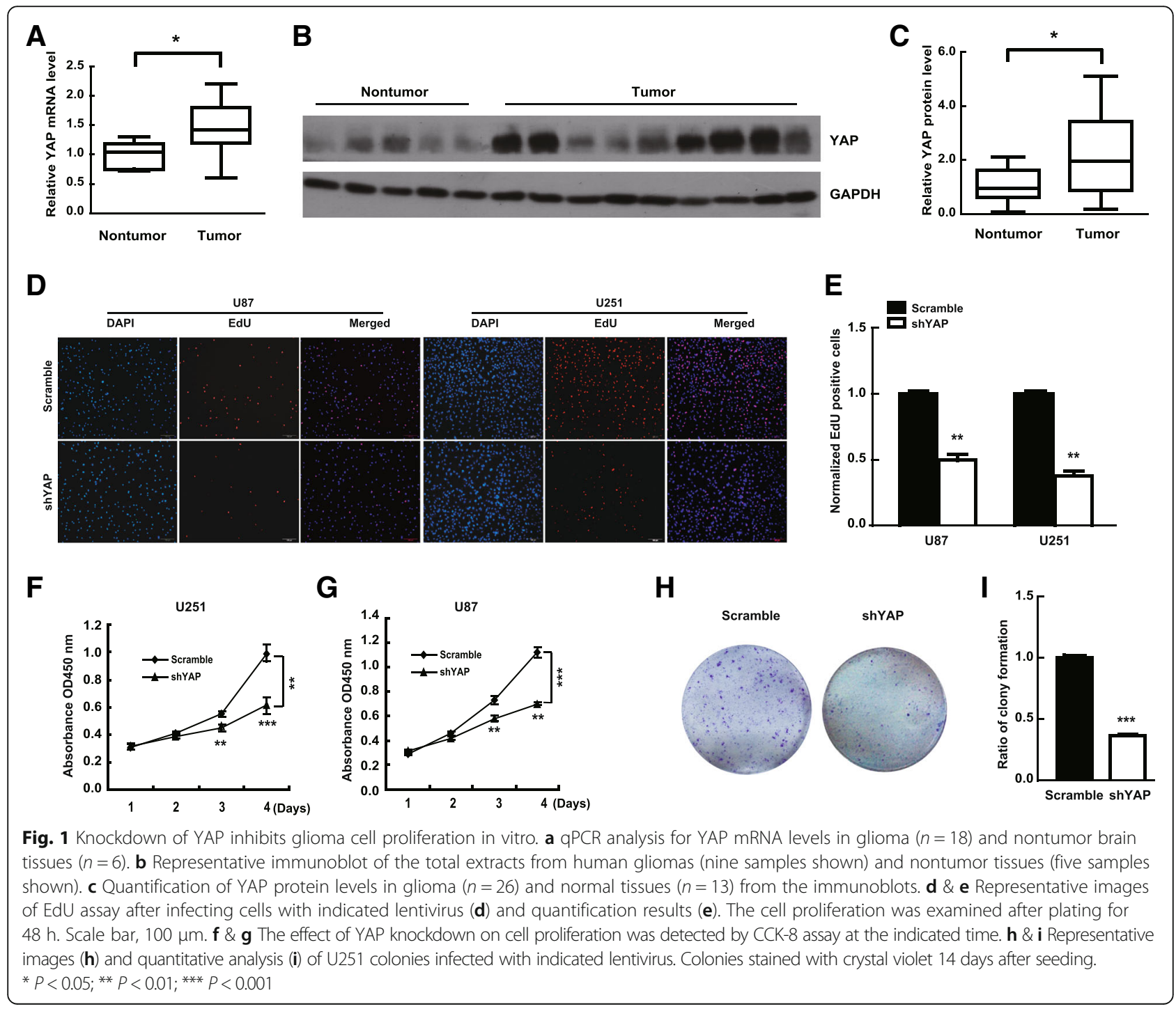


transplanted into the right striatum of nude mice. We found that the tumors derived from YAP downregulation cells were significantly smaller than those of the scramble mice (Fig. 2a, b). In addition, the median survival time was prolonged from 34 days to 46 days after YAP down-regulation (Fig. 2c), indicating that YAP down-regulation mice had a clear survival advantage. The number of mitotic cells and Ki67 positive cells of YAP-down regulation group decreased significantly (Fig. 2d-g). Interestingly, the cell density (GFP positive cells) of tumors derived from YAP down-regulation group was significantly lower than that of scramble group (Fig. 2f). The above results indicated that down-regulation of YAP inhibited intracranial glioma growth in vivo.

YAP modulates the protein level of $\beta$-catenin via regulating the activity of GSK3 $\beta$ in destruction complex

Next, we address how YAP regulates glioma progression. Hippo/YAP pathway is closely related to the $\mathrm{Wnt} / \mathrm{\beta}$-catenin signaling $[24,25]$. In addition, we and others have reported that $\mathrm{Wnt} / \beta$-catenin plays important roles in glioma development $[13-15,26]$. Whether YAP regulates $\beta$-catenin in human gliomas? We examined the protein and mRNA levels of $\beta$-catenin after YAP down-regulation or over-expression. YAP over-expression U87 glioma cells with about 90\% GFP-positive percentage were established (Additional file 2: Figure S2A and B). The protein level of $\beta$-catenin decreased after YAP down-regulation, while it increased after YAP over-expression (Fig. 3a). However, the mRNA level of $\beta$-catenin showed no change (Fig. 3b-d), in line with the previous report [21].

As the protein level of $\beta$-catenin is mainly modulated via phosphorylation of GSK3 $\beta$ in AXIN/APC/GSK3 $\beta$ destruction complex $[10,11]$, we therefore wonder whether YAP modulates the protein level of $\beta$-catenin via regulating the activity of GSK3 $\beta$ in destruction complex. As shown in Fig. $3 \mathrm{e}-\mathrm{g}$, we found that the protein levels of $\mathrm{p}-\mathrm{GSK}-3 \beta$, $\beta$-catenin and active- $\beta$-catenin decreased when YAP was down-regulated in U87 and U251 cells. On the contrary, the above three proteins increased and $p-\beta$ catenin level decreased after YAP over-expression in U87 cells (Fig. 3h, i). Interestingly, YAP S94A, a YAP mutation in the TEAD-binding domain, recapitulated the effect of YAP wild type on GSK3 $\beta$ and $\beta$-catenin phosphorylation (Additional file 3: Figure S3A and B), suggesting that YAP regulates GSK3 $\beta$ activity in the cytoplasm and the transcriptional activity of YAP is dispensable for the observed phenotypes.

\section{YAP modulates the subcellular location and transcription activity of $\beta$-catenin}

Next, we examined whether YAP affects the subcellular location and activity of $\beta$-catenin. As shown in Fig. $4 \mathrm{a}$ and $\mathrm{b}$, the fluorescence intensity (the protein level) of $\beta$-catenin and active- $\beta$-catenin decreased
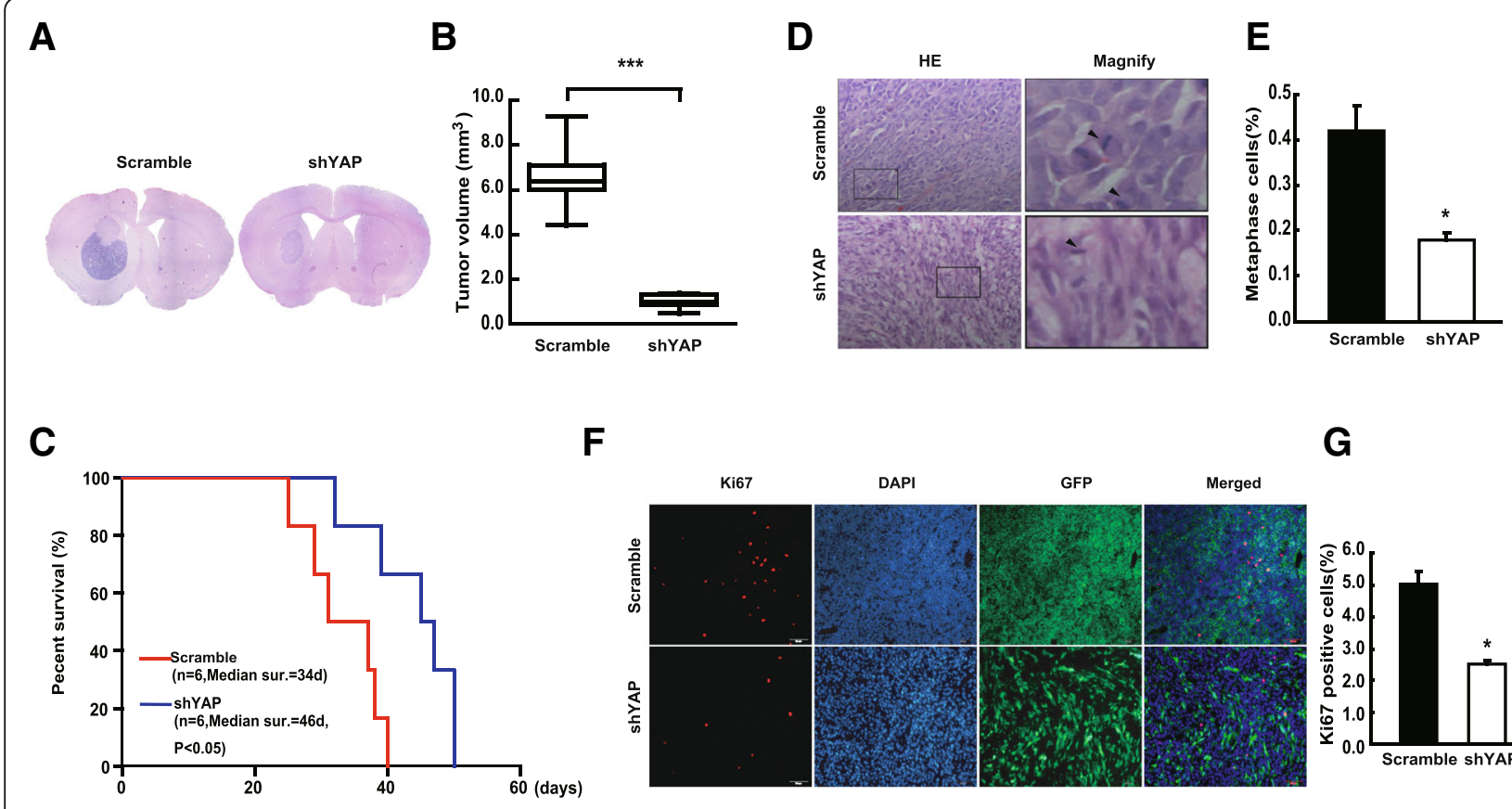

$\mathbf{F}$
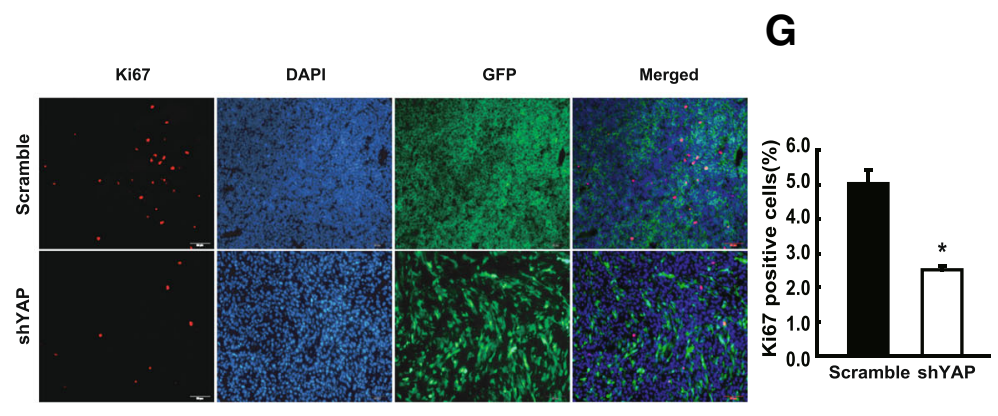

Fig. 2 Down-regulation of YAP inhibits intracranial glioma growth in vivo. a HE staining of tumors derived from scramble and shYAP cells. b Quantitative analysis of the tumor volume. c Kaplan-Meier curve of scramble and shYAP mice showed a clear survival advantage for YAP down-regulation ( $n=6$; $P<0.05$, determined using log-rank test). $\mathbf{d} \& \mathbf{e}$ The number of metaphase cells was counted per 20 high-power fields (d, right: amplified images) and quantified (e). Scale bar: $20 \mu \mathrm{m}$. f \& g Ki67 positive cells were determined by Ki67 staining. Scale bar: $50 \mu \mathrm{m}$. ${ }^{*} P<0.05,{ }^{* *} P<0.01$ 


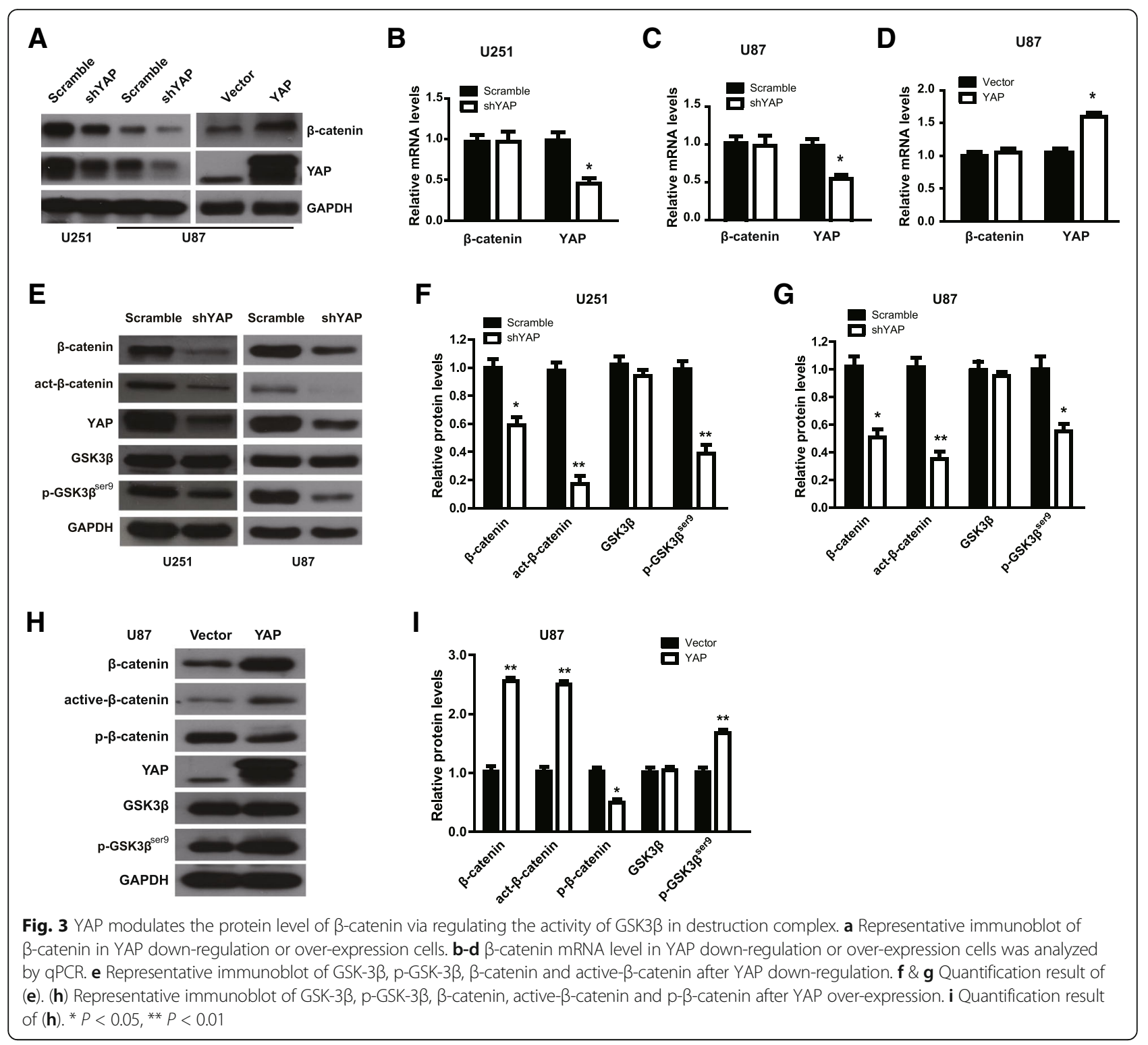

after YAP down-regulation, while it increased after YAP over-expression. Importantly, the subcellular location of the above two proteins was mainly at cytoplasm, cell membrane and the cellular junctions after YAP downregulation. Interestingly, after YAP over-expression, the above two proteins were mainly located at the nucleus (Fig. 4a and b). The percentage of cells with nuclear $\beta$ catenin and active $\beta$-catenin increased significantly after YAP over-expression, while it decreased after YAP downregulation (Fig. 4c-f). In addition, by using cellular fractionation and immunoblotting, we found that the nuclear $\beta$-catenin and active $\beta$-catenin levels increased after overexpressing YAP in U251 or U87 cells (Fig. $4 \mathrm{~g}$ and $\mathrm{h}$ ). The above results indicate that YAP overexpression increases both $\beta$-catenin protein level and nucleus translocation.
As $\beta$-catenin functions as a transcriptional co-activator to promote cell proliferation after being transported into the nucleus $[10,11]$, we guess that the increased $\beta$-catenin and active- $\beta$-catenin in the nucleus after YAP over-expression may promote the transcription of $\beta$-catenin target genes. As expected, the mRNA levels of c-myc and cyclin D1, two $\beta$-catenin target genes, decreased after YAP downregulation (Fig. $5 \mathrm{a}-\mathrm{C}$ ), while they increased after YAP overexpression (Fig. 5a and d). Together, the above results indicate that YAP enhances the transcription activity of $\beta$ catenin in glioma cells.

The effect of YAP down-regulation on glioma cell proliferation was partially mediated by $\beta$-catenin

Next, we wonder whether $\beta$-catenin mediates the effect of YAP on glioma cell proliferation. We took advantage 


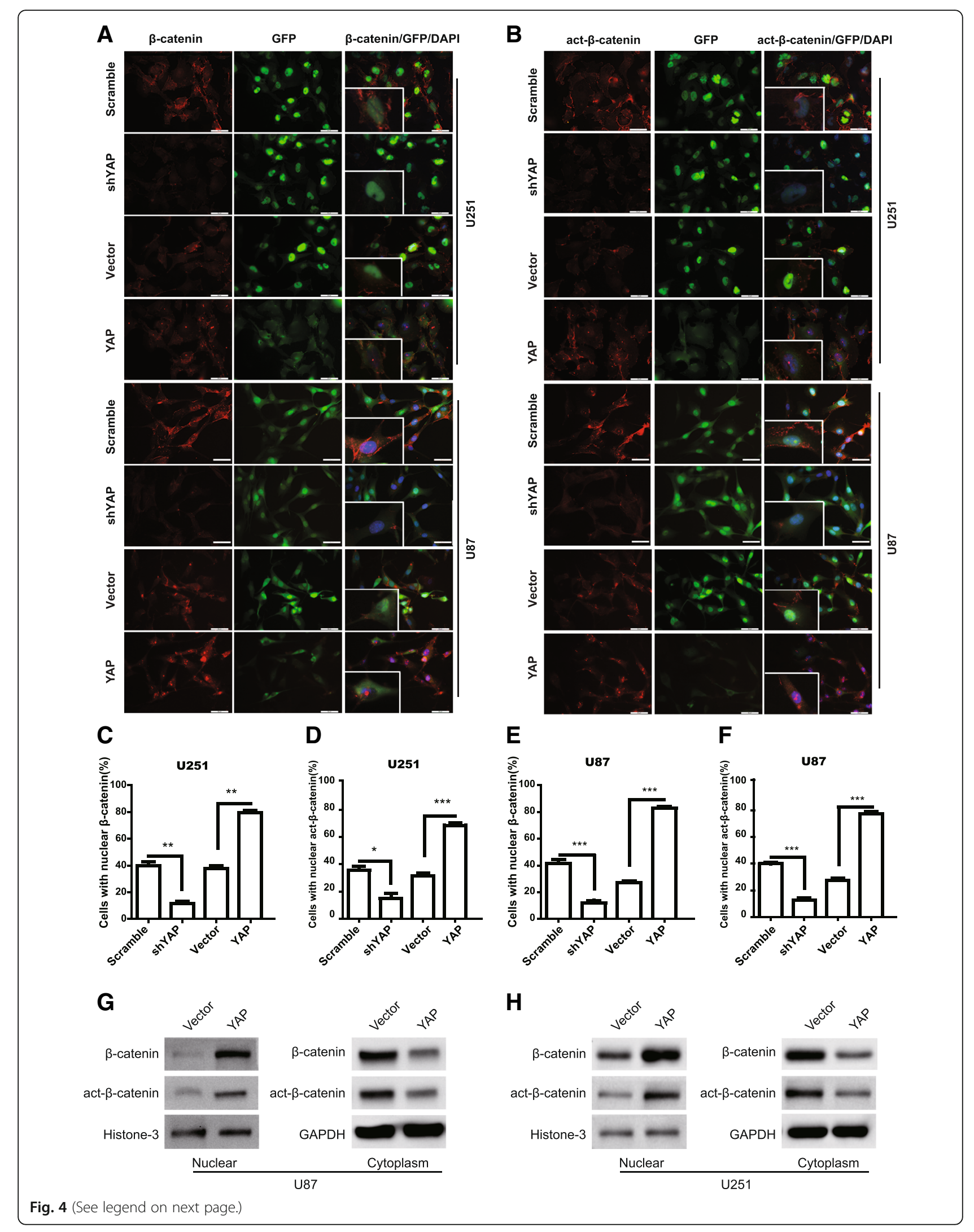


(See figure on previous page.)

Fig. 4 YAP modulates the subcellular location of $\beta$-catenin. a \& b The expression and subcellular location of $\beta$-catenin (a) and active- $\beta$-catenin (b) were assessed by immunofluorescence in YAP down-regulation or over-expression cells. Scale bar $50 \mu \mathrm{m}$. Inset showed the amplified images. c-f Quantification results of the percentage of cells with nuclear $\beta$-catenin (c \& e) or active- $\beta$-catenin $(\mathbf{d} \& \mathbf{f})$ in $\cup 251$ and $\cup 87$ cells. ${ }^{*} P<0.05$, ${ }^{* *} P<0.01$, ${ }^{* * *} P<0.001$. $\mathbf{g} \& \mathbf{h}$ Subcellular location of $\beta$-catenin or active- $\beta$-catenin was detected by using cellular fractionation and immunoblotting. Histone and GAPDH were used as nuclear and cytoplasm loading control respectively

of $\beta$-catenin ${ }^{\mathrm{WT}}$ and $\beta$-catenin ${ }^{\mathrm{CA}}$, the active form of $\beta$ catenin, to address this question. $\beta$-catenin ${ }^{\mathrm{CA}}$ is the mutated form of $\beta$-catenin with four serine/threonine residues (Ser33, Ser37, Thr41, and Ser45) changed to alanine, which cannot be phosphorylated by GSK3 $\beta$ and exhibits constitutive stabilization [27, 28]. As shown in Fig. 6a-d, both transient over-expressing $\beta$-catenin ${ }^{\mathrm{WT}}$ and its active form $\beta$-catenin ${ }^{\mathrm{CA}}$ in U251 and U87 glioma cells promoted glioma cell proliferation and $\beta$-catenin ${ }^{\mathrm{CA}}$ exhibited a stronger promotion effect than $\beta$-catenin ${ }^{\mathrm{WT}}$. In line with our previous results shown in Figs. 1 and 2, down-regulation of YAP inhibited glioma cell proliferation (Fig. 6a-d). Interestingly, when $\beta$-catenin ${ }^{\mathrm{WT}}$ and $\beta$-catenin ${ }^{\mathrm{CA}}$ were over-expressed in the cells with YAP depletion, the reduced proliferation capacity of glioma cells induced by YAP down-regulation could be partially rescued. Especially, $\beta$-catenin ${ }^{\mathrm{CA}}$ exhibited a stronger rescue effect than that of $\beta$-catenin ${ }^{\text {WT }}$ (Fig. 6a-d). These results demonstrate that YAP increases the protein level and activity of $\beta$-catenin via inhibiting the activity of GSK3 3 , which ultimately promotes the proliferation of glioma cells.

\section{Discussion}

The Hippo/YAP pathway plays a key role in regulating organ size, tissue homeostasis, and patterning $[29,30]$. Numerous studies reported that YAP is up-regulated in many types of human cancers and exhibits positive association with patient prognosis [3]. However, YAP also is reported to be down-regulated in some human tumors, such as breast cancer, hematological cancers [31, 32], indicating that YAP may exhibit a tissue- or context-dependent role in tumor biology. In our previous study, we reported that up-regulated YAP1 in human gliomas is positively associated with glioma patient prognosis and transient over-expression of YAP promotes glioma cell proliferation [8]. In the current study, we found that down-regulation of YAP inhibited glioma cell proliferation both in vitro and in vivo, indicating that YAP may be a potential molecular target for glioma treatment.

We found that YAP over-expression increased $\beta$ catenin protein level in human gliomas, consistent with the previous report [18]. However, two groups reported that shYAP1 or conditional knockout YAP did not affect
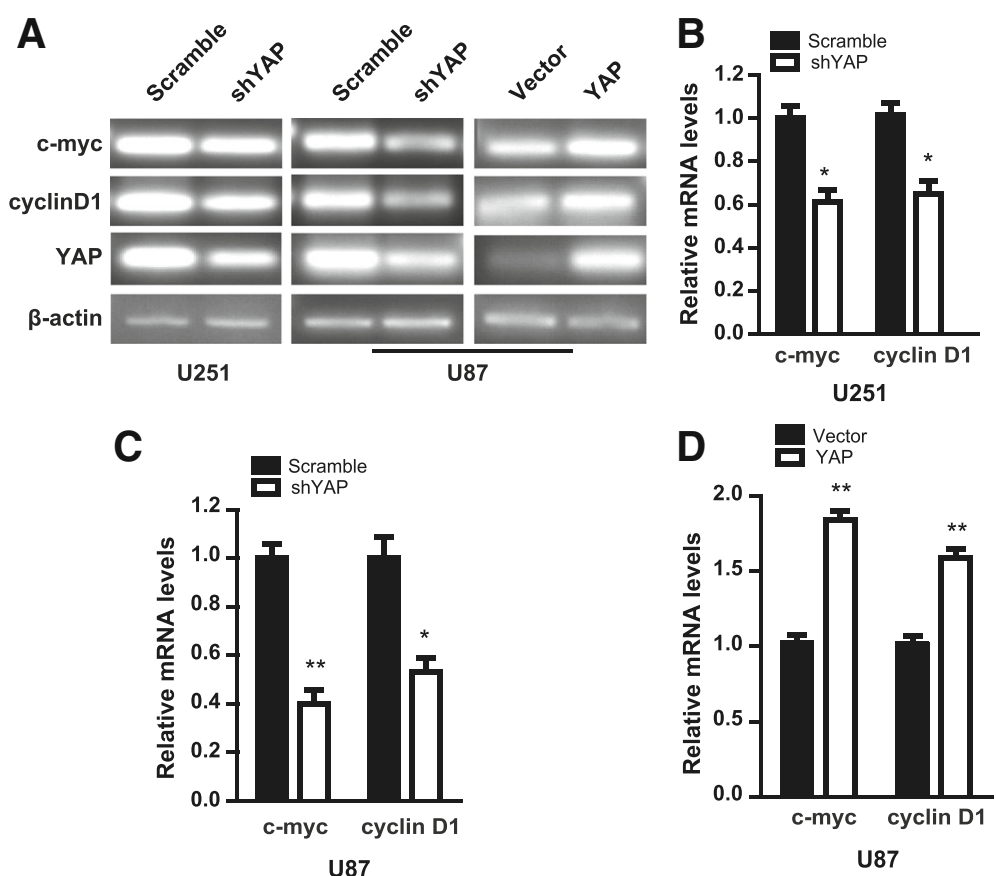

Fig. 5 YAP modulates the transcription activity of $\beta$-catenin. a c-myc and cyclin D1 expression levels were examined in YAP down-regulation or over-expression cells by RT-PCR. b-d Quantification result of $(\mathbf{a}) .{ }^{*} P<0.05,{ }^{* *} P<0.01$ 


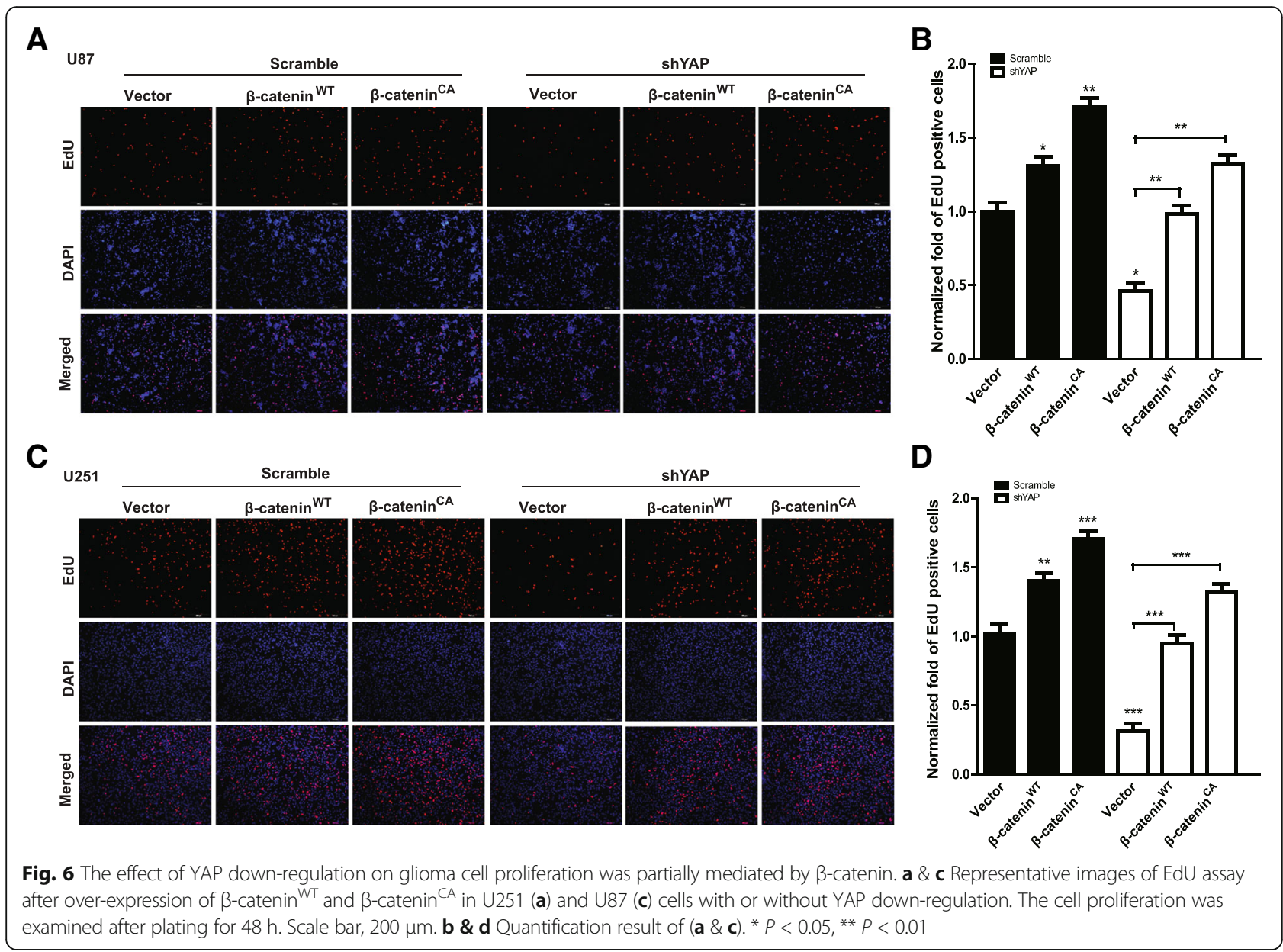

$\beta$-catenin stability or levels $[33,34]$. In our system, we found that neither down-regulation nor over-expression of YAP changed the mRNA level of $\beta$-catenin. In line with our finding, Park et al. reported that YAP/TAZ has no effect on the mRNA level of $\beta$-catenin and alternatively they are the downstream effectors of Wnt signaling [21]. The difference between above studies may be caused by different experiment system used or different functions of YAP/TAZ in different tissues. For example, the level of YAP decreased in breast cancer and in hematological cancers $[31,32]$, while it is up-regulated in human gliomas [8]. In addition, YAP, as a component of the $\beta$-catenin destruction complex, acts as $\beta$-catenin inhibitor in the WNT-OFF state and as Wnt transducer in the WNT-ON state [20]. However, in the current study, we found that YAP over-expression increased $\beta$ catenin level, nucleus translocation and transcription activity, indicating that YAP acts as a $\beta$-catenin promoter, but not an inhibitor in glioma context.

We found that the protein level of $\mathrm{p}-\mathrm{GSK}-3 \beta$ increased after YAP over-expression, leading to $\beta$-catenin and active$\beta$-catenin increase. In addition, $\beta$-catenin ${ }^{C A}$ has a stronger rescue effect than that of $\beta$-catenin ${ }^{\mathrm{WT}}$ on cell growth inhibition induced by YAP depletion, indicating that YAP inhibits GSK3 $\beta$ activity indeed. How does YAP affect the activity of GSK3 $\beta$ ? Xin. et al. reported that, in YAP ${ }^{\mathrm{S} 112 \mathrm{~A}}$ (a mouse constitutively active form of YAP and localized at the nucleus)-expressing cells, activated YAP enhances IGF signaling by phosphorylating AKT and GSK-3 $\beta$, leading to GSK-3 $\beta$ activity inhibition. Consistently, the level of $\beta$ catenin and the active non-phosphorylated $\beta$-catenin increased in $\mathrm{YAP}^{\mathrm{S} 112 \mathrm{~A}}$-expressing cardiomyocytes [35]. In 2014, Azzolin et al. reported that, in Wnt-OFF cells, although down-regulation of YAP does not affect the formation of AXIN/APC/GSK3 $\beta$ complex, it inhibits $\beta$-TrCP recruitment, leading to $\beta$-catenin activation [20]. In our study, YAP S94A, a human YAP mutation in the TEADbinding domain, recapitulated the effect of YAP wild type on GSK3 $\beta$ and $\beta$-catenin phosphorylation (Additional file 1: Figure S3A and B), suggesting that YAP regulates GSK3 $\beta$ activity in the cytoplasm and the transcriptional activity of YAP is dispensable for the observed phenotypes.

We observed that, after YAP over-expression, the increased $\beta$-catenin and active- $\beta$-catenin translocated into the nucleus, leading to c-myc and cyclin D1 transcription. As nuclear accumulation of $\beta$-catenin participates in 
malignant progression of gliomas and implicates poor prognosis [36], we deduce that the nucleus increased $\beta$-catenin and active- $\beta$-catenin after YAP over-expression may be related to the incidence and development of gliomas. Interestingly, over-expressed YAP (GFP-YAP) is mainly concentrated in the cytoplasm in the glioma cells (Fig. 4a, Additional file 2: Figure S2A) and $\beta$-catenin was mainly located in cytomembrane and the cellular junctions (Fig. 4a and $b$ ), how does over-expression of YAP promote $\beta$ catenin into the nucleus? Imajo. et al. demonstrated that phosphorylated YAP suppresses nuclear translocation of $\beta$ catenin by directly binding to it in the cytoplasm [18]. In Wnt-ON cells, release of YAP from the AXIN/APC/GSK3 $\beta$ complex promotes both YAP and $\beta$-catenin into nucleus [20]. However, both studies cannot explain our findings, in which YAP is accumulated in the cytoplasm and $\beta$-catenin in the nucleus. Whether over-expressed YAP competes with $\beta$-catenin for binding AXIN/APC/GSK3 $\beta$ complex and then releases $\beta$-catenin from the complex in glioma context? The mechanism that $\beta$-catenin translocation between the nucleus and cytoplasm after YAP overexpression in glioma cells need to be explored in the future.

\section{Conclusion}

We found that knockdown of YAP inhibited glioma cell proliferation in vitro and tumor growth in vivo. Our results identify a novel mechanism that YAP modulates the protein level, subcellular location and transcription activity of $\beta$-catenin via regulating the activity of GSK3 $\beta$ in destruction complex. $\beta$-catenin mediates the promoting effect of YAP on glioma cell proliferation, which provides a new crosstalk mechanism between Hippo/ YAP and $W n t / \beta$-catenin pathways and suggests a new strategy for human gliomas treatment.

\section{Ethics approval and consent to participate}

The use of human tissues was approved by the Ethics Committee of the Affiliated Hospital of Xuzhou Medical University (No: xyfylw2014002). Written informed consent was obtained from each patients. All animal experiments were performed according to the guidelines for the care and use of laboratory animals and were approved by IACUC of Xuzhou Medical University (No: 201,647).

\section{Additional files}

Additional file 1: Figure S1. Generation of YAP down-regulation U87 and U251 cells. (A) The infection efficiency of scramble and shYAP lentivirus in U251 and U87 cells. U87 and U251 glioma cells were infected with viral supernatant and the infection efficacy was evaluated by GFP positive cells $72 \mathrm{~h}$ later. Scale bar, $100 \mu \mathrm{m}$. PH: Phase contrast. (B) Representative immunoblots of protein extraction from shYAP infected U251 and U87 glioma cells (up panel)and YAP protein levels were quantified (bottom panel). *** $P<0.001$. (PDF $358 \mathrm{~kb}$ )
Additional file 2: Figure S2. Generation of YAP over-expression U87 cells. (A) The infection efficiency of vector and YAP lentivirus in U87 cells.U87 glioma cells were infected with viral supernatant and the infection efficacy was evaluated by GFP positive cells $72 \mathrm{~h}$ later.Scale bar, $100 \mu \mathrm{m}$. PH: Phase contrast. (B) Expression analysis of YAP protein levels by Western blotting in YAP over-expression U87 glioma cells. (PDF $90 \mathrm{~kb}$ )

Additional file 3: Figure S3. The effect of YAP on GSK-3 $\beta$ and $\beta$ catenin phosphorylation. (A\&B) Representative immunoblots of GSK-3 $\beta$, p-GSK-3 $\beta, \beta$-catenin, active- $\beta$-catenin and $p$ - $\beta$-catenin after overexpression of YAP or YAP S94A in U251 (A) and U87 (B) glioma cells.The results indicate that YAP S94A recapitulated the effect of YAP wild type on GSK3 $\beta$ and

$\beta$-catenin phosphorylation. (PDF $153 \mathrm{~kb}$ )

\section{Acknowledgments}

We thank Jiale Fu for preparing the reagent.

\section{Funding}

The research was supported by National Natural Science Foundation of China (No.81372699; No. 81472345; No. 81672489); 333 talent project of Jiangsu Province (No. BRA2015394); Six major talent summit of Jiangsu Province (No. WSW-039).

\section{Availability of data and materials}

All data used in this study are included within the article and additional files.

\section{Authors' contributions}

YW, PP and XZ conceived and designed the experiments, performed the experiments and analyzed the data and wrote the manuscript. ZW, YZ, PX, DG and YJ performed the experiments and analyzed the data. RY, YW and $X Z$ collected and analyzed clinic samples. All authors read and approved the final manuscript.

Consent for publication

Not applicable.

\section{Competing interests}

The authors declare that they have no competing interests.

\section{Publisher's Note}

Springer Nature remains neutral with regard to jurisdictional claims in published maps and institutional affiliations.

\section{Author details}

${ }^{1}$ Insititute of Nervous System Diseases, Xuzhou Medical University, 84 West Huai-hai Road, Xuzhou, Jiangsu 221002, People's Republic of China. ${ }^{2}$ Brain Hospital, Affiliated Hospital of Xuzhou Medical University, Xuzhou, Jiangsu, China. ${ }^{3}$ The Graduate School, Xuzhou Medical University, Xuzhou, Jiangsu, China. ${ }^{4}$ Present address: Department of Neurosurgery, Xuzhou Cancer Hospital, Xuzhou, Jiangsu, China. ${ }^{5}$ Jiangsu Center for the Collaboration and Innovation of Cancer Biotherapy, Cancer Institute, Xuzhou Medical University, Xuzhou, Jiangsu, China.

Received: 13 May 2017 Accepted: 22 September 2017 Published online: 29 September 2017

References

1. Wen PY, Kesari S. Malignant gliomas in adults. N Engl J Med. 2008;359:492-507.

2. Pan D. The hippo signaling pathway in development and cancer. Dev Cell. 2010;19:491-505.

3. Yu FX, Zhao B, Guan KL. Hippo Pathway in Organ Size Control, Tissue Homeostasis, and Cancer. Cell. 2015;163:811-28.

4. Harvey KF, Zhang X, Thomas DM. The Hippo pathway and human cancer. Nat Rev Cancer. 2013;13:246-57.

5. Steinhardt AA, Gayyed MF, Klein AP, Dong J, Maitra A, Pan D, Montgomery EA, Anders RA. Expression of Yes-associated protein in common solid tumors. Hum Pathol. 2008;39:1582-9. 
6. Sudol M, Shields DC, Farooq A. Structures of YAP protein domains reveal promising targets for development of new cancer drugs. Semin Cell Dev Biol. 2012;23:827-33

7. Orr BA, Bai H, Odia Y, Jain D, Anders RA, Eberhart CG. Yes-associated protein 1 is widely expressed in human brain tumors and promotes glioblastoma growth. J Neuropathol Exp Neurol. 2011;70:568-77.

8. Z Zhang H, Geng D, Gao J, Qi Y, Shi Y, Wang Y, Jiang Y, Zhang Y, Fu J, Dong Y, Gao S, Yu R, Zhou X. Expression and significance of Hippo/YAP signaling in glioma progression. Tumour Biol. 2016. [Epub ahead of print]

9. Chao Y, Wang Y, Liu X, Ma P, Shi Y, Gao J, Shi Q, Hu J, Yu R, Zhou X. Mst1 regulates glioma cell proliferation via the AKT/mTOR signaling pathway. J Neuro-Oncol. 2015;121:279-88.

10. Clevers H, Nusse R. Wnt/beta-catenin signaling and disease. Cell. 2012;149:1192-205.

11. Saito-Diaz K, Chen TW, Wang X, Thorne CA, Wallace HA, Page-McCaw A, Lee E. The way Wnt works: components and mechanism. Growth Factors. 2013;31:1-31.

12. Anastas JN, Moon RT. WNT signalling pathways as therapeutic targets in cancer. Nat Rev Cancer. 2013;13:11-26.

13. Liu $X$, Wang L, Zhao S, Ji X, Luo Y, Ling F. beta-Catenin overexpression in malignant glioma and its role in proliferation and apoptosis in glioblastma cells. Med Oncol. 2011;28:608-14.

14. Pu P, Zhang Z, Kang C, Jiang R, Jia Z, Wang G, Jiang H. Downregulation of Wnt2 and beta-catenin by siRNA suppresses malignant glioma cell growth. Cancer Gene Ther. 2009:16:351-61.

15. Zhang K, Zhang J, Han L, Pu P, Kang C. Wnt/beta-catenin signaling in glioma. J Neuroimmune Pharmacol. 2012;7:740-9.

16. Varelas X, Miller BW, Sopko R, Song S, Gregorieff A, Fellouse FA, Sakuma R, Pawson T, Hunziker W, McNeill H, Wrana JL, Attisano L. The Hippo pathway regulates Wnt/beta-catenin signaling. Dev Cell. 2010;18:579-91.

17. Heallen T, Zhang M, Wang J, Bonilla-Claudio M, Klysik E, Johnson RL, Martin JF. Hippo pathway inhibits Wnt signaling to restrain cardiomyocyte proliferation and heart size. Science. 2011;332:458-61.

18. Imajo M, Miyatake $K$, limura A, Miyamoto A, Nishida E. A molecular mechanism that links Hippo signalling to the inhibition of Wnt/beta-catenin signalling. EMBO J. 2012;31:1109-22.

19. Konsavage WM Jr, Kyler SL, Rennoll SA, Jin G, Yochum GS. Wnt/beta-catenin signaling regulates Yes-associated protein (YAP) gene expression in colorectal carcinoma cells. J Biol Chem. 2012;287:11730-9.

20. Azzolin L, Panciera T, Soligo S, Enzo E, Bicciato S, Dupont S, Bresolin S, Frasson C, Basso G, Guzzardo V, Fassina A, Cordenonsi M, Piccolo S. YAP/ TAZ incorporation in the beta-catenin destruction complex orchestrates the Wnt response. Cell. 2014;158:157-70.

21. Park HW, Kim YC, Yu B, Moroishi T, Mo JS, Plouffe SW, Meng Z, Lin KC, Yu FX, Alexander CM, Wang CY, Guan KL. Alternative Wnt Signaling Activates YAP/TAZ. Cell. 2015;162:780-94.

22. Liu X, Lu D, Ma P, Liu H, Cao Y, Sang B, Zhu X, Shi Q, Hu J, Yu R, Zhou X. Hugl-1 inhibits glioma cell growth in intracranial model. J Neuro-Oncol. 2015:125:113-21.

23. Zhou X, Xue P, Yang M, Shi H, Lu D, Wang Z, Shi Q, Hu J, Xie S, Zhan W, Yu R. Protein kinase D2 promotes the proliferation of glioma cells by regulating Golgi phosphoprotein 3. Cancer Lett. 2014;355:121-9.

24. Bernascone I, Martin-Belmonte F. Crossroads of Wnt and Hippo in epithelial tissues. Trends Cell Biol. 2013;23:380-9.

25. Wang Z, Ye J, Deng Y, Yan Z, Denduluri S, He TC. Wnt versus Hippo: A balanced act or dynamic duo? Genes Dis. 2014;1:127-8.

26. Nie E, Zhang X, Xie S, Shi Q, Hu J, Meng Q, Zhou X, Yu R. Beta-catenin is involved in Bex2 down-regulation induced glioma cell invasion/migration inhibition. Biochem Biophys Res Commun. 2015;456:494-9.

27. Liu C, Li Y, Semenov M, Han C, Baeg GH, Tan Y, Zhang Z, Lin X, He X. Control of beta-catenin phosphorylation/degradation by a dual-kinase mechanism. Cell. 2002;108:837-47.

28. Wang J, Ruan NJ, Qian L, Lei WL, Chen F, Luo ZG. Wnt/beta-catenin signaling suppresses Rapsyn expression and inhibits acetylcholine receptor clustering at the neuromuscular junction. J Biol Chem. 2008:283:21668-75

29. Ma Y, Yang Y, Wang F, Wei Q, Qin H. Hippo-YAP signaling pathway: A new paradigm for cancer therapy. Int J Cancer. 2015;137:2275-86.

30. Mo JS, Park HW, Guan KL. The Hippo signaling pathway in stem cell biology and cancer. EMBO Rep. 2014:15:642-56.

31. Cottini F, Hideshima T, Xu C, Sattler M, Dori M, Agnelli L, ten Hacken E, Bertilaccio MT, Antonini E, Neri A, Ponzoni M, Marcatti M, Richardson PG, Carrasco R, Kimmelman AC, Wong KK, Caligaris-Cappio F, Blandino G, Kuehl
WM, Anderson KC, Tonon G. Rescue of Hippo coactivator YAP1 triggers DNA damage-induced apoptosis in hematological cancers. Nat Med. 2014;20:599-606.

32. Yuan M, Tomlinson V, Lara R, Holliday D, Chelala C, Harada T, Gangeswaran R, Manson-Bishop C, Smith P, Danovi SA, Pardo O, Crook T, Mein CA, Lemoine NR, Jones LJ, Basu S. Yes-associated protein (YAP) functions as a tumor suppressor in breast. Cell Death Differ. 2008;15:1752-9.

33. Rosenbluh J, Nijhawan D, Cox AG, Li X, Neal JT, Schafer EJ, Zack TI, Wang X, Tsherniak A, Schinzel AC, Shao DD, Schumacher SE, Weir BA, Vazquez F, Cowley GS, Root DE, Mesirov JP, Beroukhim R, Kuo CJ, Goessling W, Hahn WC. beta-Catenin-driven cancers require a YAP1 transcriptional complex for survival and tumorigenesis. Cell. 2012;151:1457-73.

34. Barry ER, Morikawa T, Butler BL, Shrestha K, de la Rosa R, Yan KS, Fuchs CS, Magness ST, Smits R, Ogino S, Kuo CJ, Camargo FD. Restriction of intestinal stem cell expansion and the regenerative response by YAP. Nature. 2013;493:106-10.

35. Xin M, Kim Y, Sutherland LB, Qi X, McAnally J, Schwartz RJ, Richardson JA, Bassel-Duby R, Olson EN. Regulation of insulin-like growth factor signaling by Yap governs cardiomyocyte proliferation and embryonic heart size. Sci Signal. 2011:4:ra70.

36. Shi Z, Qian X, Li L, Zhang J, Zhu S, Zhu J, Chen L, Zhang K, Han L, Yu S, Pu $P$, Jiang $T$, Kang C. Nuclear translocation of beta-catenin is essential for glioma cell survival. J Neuroimmune Pharmacol. 2012;7:892-903.

\section{Submit your next manuscript to BioMed Central and we will help you at every step:}

- We accept pre-submission inquiries

- Our selector tool helps you to find the most relevant journal

- We provide round the clock customer support

- Convenient online submission

- Thorough peer review

- Inclusion in PubMed and all major indexing services

- Maximum visibility for your research

Submit your manuscript at www.biomedcentral.com/submit
) Biomed Central 